\title{
EFFECTS OF NUTRIENT SOURCES ON THE EARLY GROWTH OF PINEAPPLE PLANTLETS (Ananas comosus (L) Merr) IN THE NURSERY
}

\author{
Solomon Olusegun Omotoso ${ }^{1}$ \\ and Ezekiel Akinkunmi Akinrinde ${ }^{2}$ \\ ${ }^{1}$ Department of Crop, Soil and Environmental Sciences \\ Ekiti State University, Ado-Ekiti, Nigeria \\ ${ }^{2}$ Department of Agronomy, University of Ibadan. \\ Correspondence author: seguntoso@yahoo.com
}

(Received May 17, 2012/Accepted September 25, 2012)

\section{A B S T R A C T}

Inadequate information on the nutrition of pineapple limits its commercial production in Nigeria. The effects of poultry manure and urea (each at 50 and $100 \mathrm{~kg} \mathrm{~N} \mathrm{ha}^{-1}$ ), as well as their 1:1 (organo-mineral) combinations at 50, 100, 150 and $200 \mathrm{~kg} \mathrm{~N} \mathrm{ha}^{-1}$, on the early (nursery) growth of pineapple plantlets. Organo-mineral fertilization at $100 \mathrm{~kg} \mathrm{~N}^{--1}$ resulted in the highest D-leaf length, number of leaves, root length and leaf area, while the highest biomass production (approx. $304 \mathrm{~g}$, just within the 300-400 $\mathrm{g}$ desirable range for transplanting to the field) was achieved at $150 \mathrm{~kg} \mathrm{~N} \mathrm{ha}^{-1}$.

Key words: D-leaf, propagule propagation, nitrogen fertilizer

\section{INTRODUCTION}

The pineapple (Ananas comosus (L.) Merr), a leading edible member of the family Bromeliaceae, is cultivated between the latitudes approximately $30^{\circ} \mathrm{N}$ and $34^{\circ} \mathrm{S}$ (Batholomew and Kadzimin, 1977), predominantly for its fruit, which is consumed fresh or as canned fruit and juice. It dominates the world trade of tropical fruits, accounting for $51 \%$ of the world global fruit market (FAO, 2008), and has gained popularity in Nigeria (due to fruit juice importation ban), ranking sixth in the list of producers on a commercial scale and with an extensive cultivation area in the tropics. However, only 5\% of this output is exported by producing countries, and as for Nigeria the fruit is not even exported commercially 
(FAO, 2010). The pineapple fruit is the only source of bromelain, a proteolytic enzyme commonly used in the pharmaceutical industry and as a meat tenderizing agent (Fougue, 1981).

A major constraint to the expansion of pineapple cultivation in Nigeria is of planting material (Heenkenda, 2003), as the multiplication rate through natural suckering (one or two suckers per plant per year) is too low (Collins, 1960). The mother plants also produce one or two suckers after harvesting at 8-10 months of growth. The inability to get planting material for the establishment of new orchards is, therefore, a major challenge the farmer. Hence, there must be a way to make the propagule grow rapidly at the nursery stage to achieve the appropriate weight desirable for transplanting to the field. This work, therefore, sought to evaluate the effectiveness of different nutrient sources on the early growth of pineapple plantlets in the nursery.

\section{MATERIAL AND METHODS}

The study was conducted in the Screen House of Ekiti State University, Ado-Ekiti (latitude $7^{\circ} 40^{\prime} \mathrm{N}$ and longitude $5^{\circ} 15$, E) between May 2003 and July 2004. Soil texture was sandy loam and its characterization showed pH 5.8 in water, $0.08 \mathrm{~g} \mathrm{~kg}^{-1} \mathrm{~N}$, $2.20 \mathrm{~g} \mathrm{~kg}^{-1}$ organic $\mathrm{C}, 3.50 \mathrm{mg} \mathrm{kg}^{-1}$ available $\mathrm{P}$ and $0.38 \mathrm{mg} \mathrm{kg}^{-1} \mathrm{~K}$.

Applications of poultry manure (PM) $\left(2.3 \% \quad \mathrm{~N}, 0.85 \% \quad \mathrm{P}_{2} \mathrm{O}_{5}, 1.02 \%\right.$ $\left.\mathrm{K}_{2} \mathrm{O}\right)$ or urea $(45.0 \% \mathrm{~N})$ (each at 50 or $100 \mathrm{~kg} \mathrm{~N} \mathrm{ha}^{-1}$ ), and 1:1 combinations of the two (as organo-mineral fertilizer, OMF) at 50, 100, 150 and $200 \mathrm{~kg} \mathrm{~N} \mathrm{ha}^{-1}$, were replicated three times in a randomized complete block design. Six-leaf-stage sweet cayenne pineapple plantlets (pregerminated pre-nursery with $40-45 \mathrm{~g}$ propagules from stumps/stems) were planted at $0.5 \times 0.5 \mathrm{~m}$ on $2 \times 3 \mathrm{~m}$ plots, corresponding to 36 plants per plot, which were separated by $1 \mathrm{~m}$ wide paths. The initial roots of the plantlets were removed at transplanting, while weeding was done manually hand hoeing at two weeks after planting. The length of the D-leaf (cm) (the longest centrally leaf on the pineapple plant) and root length $(\mathrm{cm})$ were measured with a meter rule, and the number of leaves was determined by counting. Leaf surface area $\left(\mathrm{cm}^{2}\right)$ was measured using AM-300 (ADC BioScentific, UK) surface area meter. All the data were collected at monthly intervals from 2 months after transplanting (MAT), except for fresh and dry weight, which were measured at 4 and 6 MAT. The collected data were subjected to Analysis of Variance (ANOVA) and the differences between treatment means were separated using Duncan's Multiple Range Test (DMRT).

\section{RESULTS AND DISCUSSION}

Pineapple plantlets' D-leaf length significantly increased with fertilizer application during the growth period (Tab. 1). Thus, urea (at $100 \mathrm{~kg} \mathrm{~N} \mathrm{ha}^{-1}$ ) increased it by $14 \%$ relative to the 
Effects of nutrient sources on the early growth...

Table 1. Effects of nutrient sources on D-leaf and root length of pineapple plantlets

\begin{tabular}{|c|c|c|c|c|c|c|}
\hline \multicolumn{2}{|c|}{ Fertilizer treatments } & \multicolumn{5}{|c|}{ Months after transplanting } \\
\hline Fertilizer sources & $\begin{array}{c}\text { rate } \\
{\left[\mathrm{kgNha}^{-1}\right]}\end{array}$ & 2 & 3 & 4 & 5 & 6 \\
\hline \multicolumn{7}{|c|}{ D-leaf length [cm] } \\
\hline Control & 0 & $10.55 d^{*}$ & $17.54 \mathrm{e}$ & $24.38 \mathrm{e}$ & $29.41 \mathrm{f}$ & $35.47 \mathrm{f}$ \\
\hline \multirow{2}{*}{ Urea } & 50 & $14.49 \mathrm{c}$ & $20.99 \mathrm{c}$ & $26.15 d$ & $31.54 d$ & 38.61de \\
\hline & 100 & $14.72 \mathrm{c}$ & $21.71 \mathrm{a}$ & $27.20 \mathrm{bc}$ & $33.44 \mathrm{bc}$ & $40.37 \mathrm{c}$ \\
\hline \multirow{2}{*}{ PM } & 50 & $10.69 \mathrm{~d}$ & $18.21 \mathrm{~d}$ & $25.58 \mathrm{~d}$ & 31.98de & $38.20 \mathrm{de}$ \\
\hline & 100 & $10.38 \mathrm{~d}$ & $18.56 \mathrm{~d}$ & $27.38 \mathrm{bc}$ & $33.45 b c$ & $39.27 \mathrm{~d}$ \\
\hline \multirow{4}{*}{ OMF } & 50 & $14.72 \mathrm{c}$ & $20.89 \mathrm{c}$ & $26.54 \mathrm{~cd}$ & $32.44 \mathrm{~cd}$ & $38.06 \mathrm{e}$ \\
\hline & 100 & $16.63 a$ & $21.41 \mathrm{~b}$ & $31.21 \mathrm{a}$ & $37.34 \mathrm{a}$ & $45.67 \mathrm{a}$ \\
\hline & 150 & $16.70 \mathrm{a}$ & $21.88 \mathrm{~b}$ & $28.99 \mathrm{~b}$ & $34.40 \mathrm{~b}$ & $41.82 \mathrm{~b}$ \\
\hline & 200 & $15.64 \mathrm{~b}$ & $20.80 \mathrm{c}$ & $26.53 \mathrm{~cd}$ & $32.60 \mathrm{~cd}$ & $38.24 \mathrm{de}$ \\
\hline $\mathrm{SE} \pm$ & & 0.09 & 0.20 & 0.30 & 0.28 & 0.34 \\
\hline \multicolumn{7}{|c|}{ Root length [cm plant $\left.{ }^{-1}\right]$} \\
\hline Control & 0 & $6.33 \mathrm{e}$ & $6.94 \mathrm{e}$ & $7.93 \mathrm{e}$ & $8.71 \mathrm{e}$ & $9.83 \mathrm{f}$ \\
\hline \multirow{2}{*}{ Urea } & 50 & $9.88 \mathrm{~d}$ & $10.76 \mathrm{~d}$ & $11.71 \mathrm{~cd}$ & $12.23 \mathrm{~d}$ & $14.03 \mathrm{de}$ \\
\hline & 100 & $14.31 \mathrm{ab}$ & $14.93 b$ & $15.53 \mathrm{ab}$ & $17.37 \mathrm{~b}$ & $18.66 b c$ \\
\hline \multirow{2}{*}{ PM } & 50 & $10.36 \mathrm{~d}$ & $10.90 \mathrm{~d}$ & $11.03 \mathrm{~d}$ & $11.83 \mathrm{~d}$ & $12.74 \mathrm{e}$ \\
\hline & 100 & $11.40 \mathrm{~cd}$ & $12.23 \mathrm{~cd}$ & $12.90 \mathrm{~cd}$ & $14.01 \mathrm{~cd}$ & $14.81 \mathrm{de}$ \\
\hline \multirow{4}{*}{ OMF } & 50 & $12.16 \mathrm{cde}$ & $12.80 \mathrm{bcd}$ & $13.70 \mathrm{bc}$ & $15.00 \mathrm{bc}$ & $16.06 \mathrm{~cd}$ \\
\hline & 100 & $15.52 \mathrm{a}$ & $17.62 \mathrm{a}$ & $17.71 \mathrm{a}$ & $21.70 \mathrm{a}$ & $24.17 \mathrm{a}$ \\
\hline & 150 & $14.33 \mathrm{ab}$ & $15.20 \mathrm{~b}$ & $16.30 \mathrm{~d}$ & $16.91 \mathrm{~b}$ & $18.32 b c$ \\
\hline & 200 & $13.37 \mathrm{abc}$ & $14.93 b$ & $17.46 \mathrm{a}$ & $17.67 \mathrm{~b}$ & $17.81 \mathrm{c}$ \\
\hline $\mathrm{SE} \pm$ & & 0.88 & 0.80 & 0.74 & 0.79 & 0.83 \\
\hline
\end{tabular}

*Means with the same letter in each column are not significantly different $(\mathrm{p}<0.05)$ using DMRT $\mathrm{PM}=$ poultry manure; $\mathrm{OMF}=$ organo-mineral fertilizer

control at $6^{\text {th }}$ month of growth. The response to poultry manure was not obvious until 4 months after transplanting (MAT). The application of OMF at $100 \mathrm{~kg} \mathrm{~N} \mathrm{ha}^{-1}$ resulted in the longest D-leaf, while higher application rates caused a decrease. It was evident that the full potential of OMF could not be expressed at early growth periods due to the slow release of the elements bound in organic form in the manure. There has been a similar report plant response to PM (Adeleye et al., 2010). Maximum root length $(24.17 \mathrm{~cm})$ was obtained the combination in which $100 \mathrm{~kg} \mathrm{~N} \mathrm{ha}^{-1}$ was applied in the form of OMF, while the lowest root length $(9.83 \mathrm{~cm})$ was in the control (measured 6 months after transplanting) (Tab. 1). Increasing $\mathrm{N}$ application rates with urea increased the root length of plantlets throughout the growth period, but $100 \mathrm{~kg} \mathrm{~N} \mathrm{ha}{ }^{-1}$ consistently gave highest values. 
The number of plantlets' leaves increased with age and fertilizer application (Tab. 2). At 6 MAT, the highest number of leaves (30) was obtained with OMF application at $100 \mathrm{~kg} \mathrm{~N} \mathrm{ha}^{-1}$. This is in agreement with the findings of Olaniyi et al., (2010) for okra. Leaf area per plant was also significantly affected by fertilizer treatment. It is obvious in Table 2 that $100 \mathrm{~kg} \mathrm{~N} \mathrm{ha}^{-1}$ from OMF application enhanced pineapple growth most in terms of leaf area $\left(180.00 \mathrm{~cm}^{2}\right)$ followed by the treatment with $150 \mathrm{~kg} \mathrm{~N} \mathrm{ha}^{-1}$ from urea, while the lowest value $\left(73.87 \mathrm{~cm}^{2}\right)$ was observed in the control plots (measured 6 months after transplanting). This is in agreement with an earlier report (Aladesanwa, 2007) on jute. Leaf area was consistently higher in the plots that received OMF throughout the whole period of growth. Application of PM alone (at $100 \mathrm{~kg} \mathrm{~N} \mathrm{ha}^{-1}$ ) gave a $57 \%$ leaf area than in the control plants at 6 MAT.

Table 2. Effects of nutrient sources on the number and area of pineapple plantlets' leaves

\begin{tabular}{|c|c|c|c|c|c|c|}
\hline \multicolumn{2}{|c|}{ Fertilizer treatments } & \multicolumn{5}{|c|}{ Months after transplanting } \\
\hline $\begin{array}{c}\text { Fertilizer } \\
\text { sources }\end{array}$ & $\begin{array}{c}\text { rate } \\
{\left[\mathrm{kg} \mathrm{N} \mathrm{ha}^{-1}\right]}\end{array}$ & 2 & 3 & 4 & 5 & 6 \\
\hline \multicolumn{7}{|c|}{ Number of leaves per plant } \\
\hline Control & 0 & $9.50 b^{*}$ & $14.73 \mathrm{c}$ & $15.33 \mathrm{c}$ & $21.00 \mathrm{c}$ & $23.67 \mathrm{c}$ \\
\hline \multirow{2}{*}{ Urea } & 50 & $10.33 \mathrm{ab}$ & $15.33 \mathrm{~b}$ & $17.67 \mathrm{bc}$ & $23.34 \mathrm{ab}$ & $25.33 \mathrm{bc}$ \\
\hline & 100 & $10.33 \mathrm{ab}$ & $17.00 \mathrm{~b}$ & $18.31 \mathrm{bc}$ & $22.67 b c$ & $26.31 \mathrm{bc}$ \\
\hline \multirow{2}{*}{ PM } & 50 & $10.33 \mathrm{ab}$ & $16.00 \mathrm{bc}$ & $17.80 \mathrm{bc}$ & $20.65 d$ & $23.33 \mathrm{c}$ \\
\hline & 100 & $10.00 \mathrm{ab}$ & $16.00 \mathrm{bc}$ & $17.32 \mathrm{bc}$ & $21.32 \mathrm{bc}$ & $24.32 b c$ \\
\hline \multirow{4}{*}{ OMF } & 50 & $10.33 \mathrm{ab}$ & $17.00 \mathrm{~b}$ & $17.33 \mathrm{bc}$ & $21.33 b c$ & $24.00 \mathrm{c}$ \\
\hline & 100 & $10.67 \mathrm{a}$ & $19.31 \mathrm{a}$ & $21.00 \mathrm{a}$ & $24.67 \mathrm{a}$ & $30.00 \mathrm{a}$ \\
\hline & 150 & $10.00 \mathrm{ab}$ & $17.67 \mathrm{~b}$ & $19.30 \mathrm{ab}$ & $24.61 \mathrm{a}$ & $24.67 \mathrm{bc}$ \\
\hline & 200 & $11.00 \mathrm{a}$ & $16.32 \mathrm{bc}$ & $17.67 \mathrm{bc}$ & $22.30 \mathrm{bc}$ & $27.67 \mathrm{ab}$ \\
\hline $\mathrm{SE} \pm$ & & 0.30 & 0.51 & 0.73 & 0.90 & 1.09 \\
\hline \multicolumn{7}{|c|}{ Leaf area $\left[\mathrm{cm}^{2}\right.$ plant $\left.^{-1}\right]$} \\
\hline Control & 0 & $64.47 \mathrm{f}$ & $66.40 \mathrm{f}$ & $67.13 \mathrm{~g}$ & $71.10 \mathrm{f}$ & $73.87 \mathrm{f}$ \\
\hline \multirow{2}{*}{ Urea } & 50 & $111.66 \mathrm{~d}$ & $115.03 \mathrm{~d}$ & $116.56 \mathrm{de}$ & $117.81 \mathrm{~d}$ & $118.70 \mathrm{~cd}$ \\
\hline & 100 & $123.61 \mathrm{~b}$ & $125.13 b$ & $132.47 \mathrm{~b}$ & $135.01 \mathrm{~b}$ & $137.84 b$ \\
\hline \multirow{2}{*}{ PM } & 50 & $95.50 \mathrm{e}$ & $97.80 \mathrm{e}$ & $101.00 \mathrm{f}$ & $103.84 \mathrm{e}$ & $105.67 \mathrm{e}$ \\
\hline & 100 & $97.43 \mathrm{e}$ & $100.60 \mathrm{e}$ & $109.10 \mathrm{e}$ & $113.24 \mathrm{~d}$ & 115.70de \\
\hline \multirow{4}{*}{$\mathrm{OMF}$} & 50 & $115.53 \mathrm{~cd}$ & $117.47 \mathrm{~cd}$ & $120.03 \mathrm{~cd}$ & $122.03 \mathrm{~cd}$ & $125.70 \mathrm{~cd}$ \\
\hline & 100 & $137.60 \mathrm{a}$ & $153.80 \mathrm{a}$ & $157.90 \mathrm{a}$ & $170.01 \mathrm{a}$ & $180.00 \mathrm{a}$ \\
\hline & 150 & $118.70 \mathrm{bcd}$ & $119.63 \mathrm{bcd}$ & $127.96 b c$ & $132.16 b c$ & $137.96 \mathrm{~b}$ \\
\hline & 200 & $116.66 \mathrm{bc}$ & $122.31 \mathrm{~cd}$ & $124.80 \mathrm{bc}$ & $126.40 \mathrm{bcd}$ & $129.40 \mathrm{bc}$ \\
\hline $\mathrm{SE} \pm$ & & 2.75 & 2.83 & 2.77 & 3.34 & 3.55 \\
\hline
\end{tabular}

*Explanation: see Table 1

$\mathrm{PM}=$ poultry manure; $\mathrm{OMF}=$ organo-mineral fertilizer; $\mathrm{MAT}-$ months after transplanting 
Effects of nutrient sources on the early growth...

Table 3. Effects of nutrient sources on mean biomass yield (g/plant) of plantlets at 4 and 6 months after transplanting (MAT)

\begin{tabular}{|l|c|c|c|c|c|}
\hline \multirow{2}{*}{$\begin{array}{c}\text { Fertilizer } \\
\text { sources }\end{array}$} & \multirow{2}{*}{$\begin{array}{c}\text { Treatment } \\
{\left[\mathrm{kg} \mathrm{N} \mathrm{ha}^{-1}\right]}\end{array}$} & \multicolumn{2}{|c|}{$\begin{array}{c}\text { Fresh weight [g/plant] } \\
\text { MAT }\end{array}$} & \multicolumn{2}{c|}{$\begin{array}{c}\text { Dry weight [g/plant] } \\
\text { MAT }\end{array}$} \\
\cline { 2 - 6 } & & 4 & 6 & 4 & 6 \\
\hline Control & 0 & $111.56 \mathrm{e}^{*}$ & $175.77 \mathrm{~d}$ & $97.40 \mathrm{~d}$ & $103.40 \mathrm{e}$ \\
\hline \multirow{2}{*}{ Urea } & 50 & $126.13 \mathrm{de}$ & $188.10 \mathrm{~cd}$ & $103.03 \mathrm{~d}$ & $106.10 \mathrm{e}$ \\
\cline { 2 - 6 } & 100 & $179.13 \mathrm{~b}$ & $252.90 \mathrm{~b}$ & $152.10 \mathrm{~b}$ & $160.83 \mathrm{c}$ \\
\hline \multirow{2}{*}{$\mathrm{PM}$} & 50 & $143.43 \mathrm{~cd}$ & $205.10 \mathrm{c}$ & 125.53 & $112.56 \mathrm{de}$ \\
\cline { 2 - 6 } & 100 & $152.86 \mathrm{c}$ & $238.53 \mathrm{~b}$ & $126.57 \mathrm{c}$ & $122.50 \mathrm{de}$ \\
\hline \multirow{3}{*}{ OMF } & 50 & $205.60 \mathrm{a}$ & $288.67 \mathrm{a}$ & $186.80 \mathrm{a}$ & $195.60 \mathrm{~b}$ \\
\cline { 2 - 6 } & 100 & $216.93 \mathrm{a}$ & $297.30 \mathrm{a}$ & $175.07 \mathrm{a}$ & $211.26 \mathrm{a}$ \\
\cline { 2 - 6 } & 150 & $222.07 \mathrm{a}$ & $304.30 \mathrm{a}$ & $192.00 \mathrm{a}$ & $212.30 \mathrm{a}$ \\
\hline \multirow{2}{*}{$\mathrm{SE} \pm$} & 200 & $143.44 \mathrm{~cd}$ & $238.60 \mathrm{~b}$ & $117.33 \mathrm{~d}$ & $132.51 \mathrm{~d}$ \\
\hline
\end{tabular}

*Explanation: see Table 1

$\mathrm{PM}=$ poultry manure, $\mathrm{OMF}=$ organo-mineral fertilizer, $\mathrm{MAT}-$ months after transplanting

Total fresh and dry weights of pineapple plantlets (Tab. 3) at 4 and 6 MAT increased significantly with the various fertilizer treatments. OMF at $150 \mathrm{~kg} \mathrm{~N}^{-1}$ gave the highest plant dry weight (212.30 g/plant), which was not significantly different from that achieved at $100 \mathrm{~kg} \mathrm{~N} \mathrm{ha}^{-1}$. The lowest value (103.40 g/plant) was recorded for the control treatment (measured 6 months after transplanting). About $160.80 \mathrm{~g} \mathrm{DM}$ plant $^{-1}$ was obtained with the addition of $100 \mathrm{~kg} \mathrm{~N} \mathrm{ha}{ }^{-1}$ from urea, while $122.5 \mathrm{~g} \mathrm{DM}$ plant $^{-1}$ was obtained at $100 \mathrm{~kg} \mathrm{~N} \mathrm{ha}^{-1}$ from PM. Urea gave a $56 \%$ and $\mathrm{PM}$ an $18 \%$ increase over the control. The same trend was observed for fresh weight. Application of OMF at $150 \mathrm{~kg} \mathrm{~N}^{-1}$ gave the highest plant fresh weight $(304.30 \mathrm{~g} /$ plant $)$, which was not significantly different from the combination in which $100 \mathrm{~kg} \mathrm{~N}^{-1}$ was applied. The lowest value of plantlet fresh weight (175.77 $\mathrm{g} /$ plant) was obtained in the control plots (measured 6 months after transplanting).

\section{CONCLUSION}

Application of the organomineral fertilizer (OMF) to supply the plants with $100 \mathrm{~kg} \mathrm{~N} \mathrm{ha}^{-1}$ resulted in the best performance of pineapple plantlets in terms of D-leaf length, number of leaves, root length and leaf area. Fresh biomass as an index of growth in pineapple was the highest at $150 \mathrm{~kg} \mathrm{~N}^{-1}$, which produced plantlets weighing over $300 \mathrm{~g}-$ a desirable size for transplanting to the field.

\section{REFERENCES}

Adeleye E.O., Ayeni L.S, Ojeniyi S.O. 2010. Effect of poultry manure on soil physico-chemical properties, leaf nutrient contents and yield of yam (Dioscorea rotundata) on alfisol in Southwestern Nigeria. J. AMERICA SCI. 6(10): 871-878. 
Aladesanwa R.D. 2007. Effects of atrazine residue and poultry manure on the growth and development of long-fruited jute (Corchorus olitorius L.) under screenhouse conditions in southwestern Nigeria. BULL. SCI. ASSOC. NIGERIA 28: 64-67.

Agboola A.A., Obigbesan G.O. 1974. The response of some improved food crop varieties to fertilizers in the forest zone of Western Nigeria. In: Report of FAO/NORAD/FAD seminar on fertilizer use development in $\mathrm{Ni}$ geria, Ibadan, $77 \mathrm{p}$.

Bartholomew D.P., Kadzimin S.B. 1977. Pineapple. In: Alvin P.T., Kozlowski T.T. (eds), Eco-physiology of Tropical Crops. Academic Press New York, pp. 113-156.

Bouyoucos G.J. 1951. A recalibration of the hydrometer method for making mechanical analyses of soil. AGRONOM. J. 43: 34-95.

Collins J.L. 1960. The pineapple. Botany, cultivation and utilization. New York, Inter Science Publishers, p. 294.
FAO 2008. Fertilizer and their use. FAO Rome Italy.

FAO 2010. FAOSTAT Database. http://www.fao.org/site 342. Date assessed 24/8/2011.

Fougue A. 1981. Les plantes medicinales presentes en forets guyanaie. FRUITS 35(9): 503-528.

Heenkenda H.M.S. 2003. Adaptability of pineapple variety to climatic and edaphic parameters in selected agroecological zones of Sri Lanka. Ph.D. Thesis, Postgraduate Institute, University of Peradeniya, $271 \mathrm{p}$.

Olaniyi J.O, Akanbi W.B., Olaniran O.A, Ilupeju O.T. 2010. The effect of organomineral and inorganic fertilizers on the growth, fruit yield, quality and chemical compositions of okra. J. ANIMAL PLANT SCI. 9(1): 1135-1140.

Selemat M.M. 1997. Effect of size of planting materials on growth, yield and fruit quality of pineapple cv. Guandul grown on deep peat soil in the humid tropics of Malaysia. ACTA HORT. 425: 377-384.

\section{WPŁYW ŹRÓDEŁ SKŁADNIKÓW ODŻYWCZYCH NA POCZAॄTKOWY WZROST SADZONEK ANANASA W SZKÓŁCE}

\section{Solomon Olusegun Omotoso i Ezekiel Akinkunmi Akinrinde}

\section{S T R E S Z C Z E N I E}

Niewystarczające informacje o odżywianiu roślin ananasa ograniczają jego produkcję na skalę handlową. Oceniano wpływ nawozu z odchodów drobiu oraz mocznika (każdy w dawce 50 i $100 \mathrm{~kg} \mathrm{~N}^{-1}$ ), jak również ich kombinacje 1:1 (organiczno-mineralne) $\mathrm{w}$ dawkach $50,100,150 \mathrm{i} 200 \mathrm{~kg} \mathrm{~N} \mathrm{ha}^{-1}$, na początkowy wzrost (w szkółce) sadzonek ananasa. Nawożenie organiczno-mineralne w dawce $100 \mathrm{~kg} \mathrm{~N} \mathrm{ha}^{-1}$ dało największą długość liścia D, liczbę liści, długość korzeni i powierzchnię liści, natomiast najwyższą produkcję biomasy (ok. 304 g, czyli już w zakresie 300-400 g odpowiednim do posadzania w polu) osiągnięto stosując dawkę $150 \mathrm{~kg} \mathrm{~N} \mathrm{ha}^{-1}$.

Słowa kluczowe: liść D, rozmnażanie przez propagule, nawóz azotowy 